\title{
Validity investigation of random energy flow analysis for beam structures
}

\author{
Jin You*, Hong-Guang Li and Guang Meng \\ State Key Laboratory of Mechanical System and Vibration, Shanghai Jiao Tong University, Shanghai, China
}

Received 31 January 2010

Revised 14 April 2010

\begin{abstract}
The validity of the application of energy flow analysis for beam structures under random excitations is investigated in this paper. The approximate solutions of energy density and intensity in a beam subject to random loadings are obtained by solving the governing equation of random energy flow analysis using Fourier transform technique. The formulations of the exact energy density distribution and intensity in the beam are derived using the classical modal analysis method. For a simply supported beam subject to distributed or concentrated random excitations, the validity of random energy flow analysis is investigated through comparisons between solutions obtained from the approximate and exact methods for energy response as well as intensity. The results indicate that, the mode count of the analysis frequency band, which means the number of modes involved in the band, is the key factor affecting the prediction accuracy of random energy flow analysis, and that if the mode count of the band is sufficiently large, random energy flow analysis can provide rather accurate estimates for both energy density and intensity in a wide frequency range.
\end{abstract}

Keywords: Random energy flow analysis (random EFA), random excitation, energy density, intensity, beam structure

\section{Introduction}

Nowadays, the prediction of the dynamic behavior of structures in the high frequency range is a problem becoming more and more important and demanding. Statistical energy analysis (SEA) method is currently the most commonly employed simulation tool for the high frequency response estimation of structural/acoustical systems [16]. In SEA, a complex system is divided into a number of coupling subsystems, and the lumped energy stored within each subsystem is the primary variable of this approach. In the application of SEA, only a linear algebraic system equation needs to be solved, thus SEA is very computationally efficient. However, since the development of the basic SEA formulation is based on some restrictive assumptions and approximations [1,7], limited confidence can be put in the predicted results by the method. Though theoretical improvements have been achieved to improve the performance of SEA in response estimation $[11,17,18]$, they apply to simple coupling structures and are difficult to be extended to general complex ones. In addition, because SEA is a lumped parameter method, which provides no information about the local variation of the dynamic state on a subsystem, this method can not be applied efficiently in situations where local features of a subsystem, such as localized excitation and damping, must be modeled.

In order to overcome limitations posed by SEA, researchers has made great efforts in the development of alternative prediction techniques, which include the wave intensity analysis (WIA) $[12,13]$ and energy flow analysis (EFA) [2, $3,10,15,24]$. In WIA, the diffuse vibration wave filed assumption, which is required in conventional SEA, is relaxed, and the directional dependence of the energy response of each subsystem is taken into account. WIA has demonstrated improved evaluation accuracy on the conventional SEA with not too much additional computation

*Corresponding author. Tel.: +86 13788938539; E-mail: youjin1017@ hotmail.com. 
effort than the latter. EFA is an analytical prediction approach based on energy variables as well, where the time averaged over a period and space averaged over a wavelength energy density constitutes the primary variable of the method. The analogy with the thermal conductivity is adopted in the development of the EFA approach, and the governing equations of the method are derived based on a local power balance and a simplified relationship relating power flow with energy density. Local response on a subsystem can be predicted by EFA, and the method can be implemented relatively easily using finite element method, thus EFA has met a high interest in the scientific community.

The basic equations of EFA are derived for single structures, such as a rod, beam, plate or membrane $[2,3$, $10,15,24]$, and the method can apply to built-up structures by utilizing the power transfer characteristics at the junctions [5,6]. At present, the application of EFA has been extended to practical engineering structures, such as a plate reinforced with multiple parallel beams [19] and plate structures in contact with dense fluid [26-28]. In other researches concerning EFA, the method is utilized in combination with other approaches to deal with specific coupling situations. A hybrid method that combines EFA with conventional FEM is proposed to model different parts of coupled structures [21,29], and another hybrid method, which combines EFA with boundary element method, is developed to compute response of coupling structural/acoustic systems [22,23]. Because the assumption, which is associated with a far-field incoherent plane wave approximation, is introduced in the derivation of the governing equations of EFA, errors can be caused in the evaluation results of the method [14]. Theoretical efforts has been made on the improvement of the EFA theory as well as its validity investigation especially for two-dimensional systems, and it is indicated that the interference among propagating waves has also contribution to the energy propagation, which is not analogous to heat conduction [4,20].

In previous development and applications of EFA, the method is commonly employed to predict the single frequency or frequency averaged energy response of structures. The energy flow characteristics of beams and plates under random excitations were once investigated using EFA [8,9], where the energy response characteristics of the structures in one frequency band in the relatively low frequency range were mainly focused on. The application of EFA for beam structures under random excitation is systematically proposed by You et al. [25], which is referred to as random energy flow analysis. In random EFA, the average energy density is not solved directly from the energy conductivity equation of EFA but indirectly obtained from the spectral integration of an energy spectrum variable, and a detailed relationship between random EFA and SEA is established with respect to both energy and power flow variables for coupled beam structures. The proposed approach is applied to a frame subject to concentrated random broadband disturbances, and good agreement between energy as well as power flow solutions is demonstrated for subsystems close to the location of excitations, based on which the validity of the random EFA application is established. However, since SEA is an approximate approach offering solutions with limited reliability, it is necessary for the validity investigation of the random EFA application to be performed based on some exact solutions of structural response. In this paper, random EFA is applied to a single beam structure subject to concentrated or distributed random excitations, and both energy density distribution and intensity in the beam are calculated. The exact energy and intensity of the beam under random excitation is obtained using classical modal superposition method. Verification of random EFA is implemented through comparisons between approximate solutions and exact results in consecutive frequency bands ranging from low to high frequencies, and meanwhile, factors affecting the prediction accuracy of random EFA for beam structures are identified.

\section{Random energy flow analysis of a single beam}

\subsection{Random EFA energy formulation}

For a beam structure subject to a transverse concentrated random excitation, the differential equation of EFA, which reflects the power balance relationship at local place on the beam, should apply to the differential spectral component of the relative energy variable and power input as

$$
-\frac{c_{g}^{2}}{\eta \omega} \frac{\partial^{2}}{\partial x^{2}}[E(x, \omega) \mathrm{d} \omega]+\eta \omega E(x, \omega) \mathrm{d} \omega=\delta\left(x-x_{0}\right) \Pi_{i n}(\omega) \mathrm{d} \omega
$$


where $E$ is an energy variable associated with position as well as frequency, which represents the time and locally space averaged energy density spectrum [25], $\omega$ is the radian frequency of the excitation, $c_{g}$ is the group velocity of bending waves, $\eta$ is the structural loss factor, $\delta(x)$ is the Dirac delta function defining the location of the driving force, $\Pi_{i n}$ is the real part of the cross spectrum between the random force and velocity response at the driving point, which is defined as the power input spectrum of the random excitation.

The time averaged energy density of waves with different frequencies are incoherent and can be superimposed, and this accounts for the validity of applying the energy conductivity equation of EFA with respect to the differential spectral component of relative spectrum quantities, as expressed in Eq. (1). Eliminating $\mathrm{d} \omega$ at both sides of Eq. (1) yields

$$
-\frac{c_{g}^{2}}{\eta \omega} \frac{\partial^{2} E(x, \omega)}{\partial x^{2}}+\eta \omega E(x, \omega)=\delta\left(x-x_{0}\right) \Pi_{i n}(\omega)
$$

The average energy density and intensity of a beam under stationary random excitation can be obtained from the integration of the approximate energy density spectrum solved from Eq. (2), which are respectively given by [25]

$$
\begin{aligned}
& e(x)=\frac{1}{2 \pi} \int_{0}^{+\infty} E(x, \omega) \mathrm{d} \omega, \\
& I(x)=-\frac{1}{2 \pi} \int_{0}^{+\infty} \frac{c_{g}^{2}}{\eta \omega} \frac{\partial E(x, \omega)}{\partial x} \mathrm{~d} \omega
\end{aligned}
$$

\subsection{Analytical solution of the random EFA energy equation for a single beam}

Though finite element method has become the standard numerical implementation of the EFA energy equation, for a single beam, in order to find the analytical solution of energy density and intensity, Fourier transform technique is utilized to solve the random EFA governing equation. An advantage of using Fourier transform technique is that both concentrated and distributed excitations can be treated in the same manner.

For a common loading situation, Eq. (2) can be written as

$$
-\frac{c_{g}^{2}}{\eta \omega} \frac{\partial^{2} E(x, \omega)}{\partial x^{2}}+\eta \omega E(x, \omega)=\Pi_{i n}(x, \omega)
$$

where $\Pi_{i n}(x, \omega)$ represents the power input spectrum of concentrated or distributed random force. Implementing Fourier transform to Eq. (5) with respect to $x$ to cast it into the wave number space yields

$$
\frac{c_{g}^{2}}{\eta \omega} k^{2} \hat{E}(k, \omega)+\eta \omega \hat{E}(k, \omega)=\hat{\Pi}_{i n}(k, \omega)
$$

where $\hat{E}(k, \omega)$ and $\hat{\Pi}_{i n}(k, \omega)$ denote the Fourier transforms of $E(x, \omega)$ and $\Pi_{i n}(x, \omega)$ with respect to position respectively. Through algebraic transformation, Eq. (6) becomes

$$
\hat{E}(k, \omega)=\hat{H}(k) \hat{\Pi}_{i n}(k, \omega)
$$

where

$$
\hat{H}(k)=\frac{\eta \omega}{(\eta \omega)^{2}+\left(c_{g} k\right)^{2}}
$$

Based on convolution theorem, $E(x, \omega)$ can be obtained from convolution between the Fourier inversion of $\hat{H}(k)$ and $\Pi_{i n}(x, \omega)$, which is given by

$$
E(x, \omega)=\int_{0}^{L} h(x-\xi) \Pi_{i n}(\xi, \omega) \mathrm{d} \xi
$$


where

$$
h(x)=\frac{1}{2 \pi} \int_{-\infty}^{+\infty} \hat{H}(k) \mathrm{e}^{\mathrm{j} k x} \mathrm{~d} k=\frac{1}{2 c_{g}} \mathrm{e}^{-\left(\eta \omega / c_{g}\right)|x|}
$$

In Eq. (9), $L$ denotes the beam length. The result given by Eq. (9) is the particular solution component of Eq. (5), and when the general solution is included, the complete solution is expressed as

$$
E(x, \omega)=\int_{0}^{L} h(x-\xi) \Pi_{i n}(\xi, \omega) \mathrm{d} \xi+A \mathrm{e}^{\left(\eta \omega / c_{g}\right) x}+B \mathrm{e}^{-\left(\eta \omega / c_{g}\right) x}
$$

The last two items in Eq. (11) correspond to the general solution part of Eq. (5) when $\Pi_{i n}(x, \omega)$ is set to zero. The two unknown coefficients $A$ and $B$ in Eq. (11) can be determined by applying energy boundary conditions at both beam ends. For a simply supported beam, the energy boundary conditions are expressed as

$$
-\left.\frac{c_{g}^{2}}{\eta \omega} \frac{\partial E}{\partial x}\right|_{x=0}=-\left.\frac{c_{g}^{2}}{\eta \omega} \frac{\partial E}{\partial x}\right|_{x=L}=0
$$

The energy density and intensity on the beam can be obtained respectively by substituting Eq. (11) into Eqs (3) and (4).

\section{Exact solution from modal analysis}

\subsection{Energy density response of a beam subject to random excitation}

The motion equation for a transversely vibrating beam under stationary random excitation is given by

$$
E_{c} I_{0} \frac{\partial^{4} u(x, t)}{\partial x^{4}}+m \frac{\partial^{2} u(x, t)}{\partial t^{2}}=f(x, t)
$$

where $u(x, t)$ is the transverse displacement, $E_{c}=E_{0}(1+\mathrm{j} \eta)$ is the complex modulus of elasticity, $\eta$ is the structural loss factor, $I_{0}$ is the cross-section inertia moment, and $m$ is the density per unit length. Through modal superposition, the equation determining the response amplitude of an arbitrary mode can be obtained as

$$
u_{n}^{\prime \prime}+\omega_{n}^{2}(1+\mathrm{j} \eta) u_{n}=\frac{1}{M} \int_{0}^{L} f(r, t) \varphi_{n}(r) \mathrm{d} r
$$

where $u_{n}, \omega_{n}$ and $\varphi_{n}$, respectively, are the amplitude, natural frequency and mode shape of the $n$th mode, and $M$ is the total mass of the beam. Performing Fourier transform to Eq. (14) for a finite time interval, $0 \leqslant t \leqslant T$, and solving for $u_{n}$, it yields

$$
U_{n}(\omega, T)=\frac{1}{M} \int_{0}^{L} \varphi_{n}(r) H_{n}(\omega) F(r, \omega, T) \mathrm{d} r
$$

where $H_{n}(\omega)=1 /\left[\omega_{n}^{2}(1+\mathrm{j} \eta)-\omega^{2}\right]$ is the $n$th transfer function of the beam, and $F$ and $U_{n}$ are the Fourier transforms of the acting force and the $n$th modal displacement respectively. The displacement response of the beam is obtained by summing the contributions of all modes as

$$
U(x, \omega, T)=\sum_{n} U_{n}(\omega, T) \varphi_{n}(x)=\int_{0}^{L}\left[\frac{1}{M} \sum_{n} \varphi_{n}(x) \varphi_{n}(r) H_{n}(\omega)\right] F(r, \omega, T) d r
$$


In Eq. (16), the order of integral and infinite summation has been changed. The Fourier transform of the velocity response can be obtained from that of the displacement response, as

$$
V(x, \omega, T)=\mathrm{j} \omega U(x, \omega, T)=\int_{0}^{L}\left[\frac{\mathrm{j} \omega}{M} \sum_{n} \varphi_{n}(x) \varphi_{n}(r) H_{n}(\omega)\right] F(r, \omega, T) \mathrm{d} r
$$

If the velocity mobility function between positions $x$ and $r$ is defined as

$$
Y(x, r, \omega)=\frac{\mathrm{j} \omega}{M} \sum_{n} \varphi_{n}(x) \varphi_{n}(r) H_{n}(\omega)
$$

Equation (17) takes the following form

$$
V(x, \omega, T)=\int_{0}^{L} Y(x, r, \omega) F(r, \omega, T) d r
$$

The auto-spectrum of velocity can be expressed in terms of the cross-spectral function of the random distributed loading between different positions, given by

$$
S_{v}(x, \omega)=\lim _{T \rightarrow \infty} \frac{1}{T} E\left[V^{*} V\right]=\int_{0}^{L} \int_{0}^{L} Y^{*}(x, r, \omega) Y(x, s, \omega) S_{f}(r, s, \omega) d r d s
$$

where

$$
S_{f}(r, s, \omega)=\lim _{T \rightarrow \infty} \frac{1}{T} E\left[F^{*}(r, \omega, T) F(s, \omega, T)\right]
$$

$S_{f}$ is the cross-spectrum of the force between positions $r$ and $s$, which is related to the cross-correlation function of the force at different locations through Fourier inversion, given by

$$
S_{f}(r, s, \omega)=\int_{-\infty}^{+\infty} R_{f}(r, s, \tau) e^{-\mathrm{j} \omega \tau} d \tau
$$

where

$$
R_{f}(r, s, \tau)=E[f(r, t) f(s, t+\tau)]
$$

is the cross-correlation function of the acting force. If a single concentrated random force at $x_{0}$ is considered, the cross-spectrum of the force takes a simple form as

$$
S_{f}(r, s, \omega)=S_{f}(\omega) \delta\left(r-x_{0}\right) \delta\left(s-x_{0}\right)
$$

Mean square value of the random velocity along the beam is calculated by the integration of its auto-spectrum, $v_{\mathrm{RMS}}^{2}=(1 / 2 \pi) \int_{-\infty}^{+\infty} S_{v}(x, \omega) \mathrm{d} \omega$, and mean square value of the displacement is obtained in the same way. The total average energy density of a beam is the sum of the average kinetic and potential energy responses, given by

$$
\bar{e}(x)=\bar{e}_{k}(x)+\bar{e}_{p}(x)=\frac{1}{2} E_{0} I_{0} \frac{\partial^{2} u_{\mathrm{RMS}}^{2}(x)}{\partial x^{2}}+\frac{1}{2} m v_{\mathrm{RMS}}^{2}(x)
$$

where the overbar indicates the time averaged quantity. 
Table 1

Properties of beam

\begin{tabular}{lc}
\hline Young's Modulus of Elasticity $\left(\mathrm{N} / \mathrm{m}^{2}\right)$ & $7.0 \mathrm{E}+10$ \\
\hline Mass Density $\left(\mathrm{kg} / \mathrm{m}^{3}\right)$ & 2700.0 \\
Structural loss factor & 0.03 \\
Length $(\mathrm{m})$ & 10 \\
Cross-sectional Dimensions, width $\times$ height $(\mathrm{m})$ & $0.025 \times 0.025$ \\
\hline
\end{tabular}

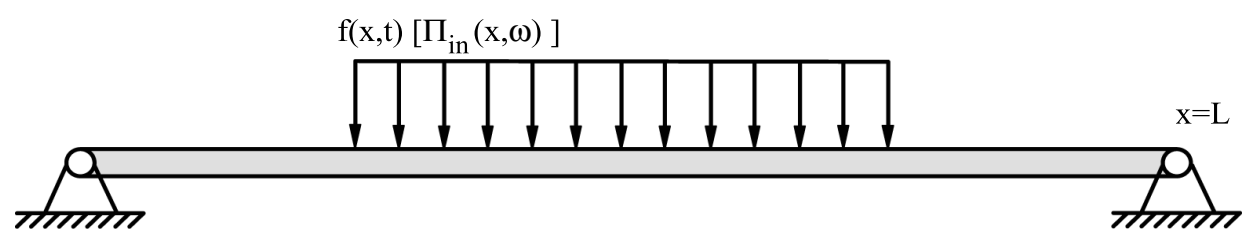

Fig. 1. A simply supported beam subject to distributed random excitation.

\subsection{Intensity in the beam}

Intensity in transversely vibrating beams is produced by two separate internal mechanisms: the shear force and the bending moment. The instantaneous power flow associated with the shear force is written in terms of the displacement and velocity as

$$
p_{\mathrm{sh}}(x, t)=E_{0} I_{0} \frac{\partial^{3} u(x, t)}{\partial x^{3}} v(x, t)
$$

The instantaneous power flow caused by the bending moment is

$$
p_{\text {mo }}(x, t)=-E_{0} I_{0} \frac{\partial^{2} u(x, t)}{\partial x^{2}} \frac{\partial v(x, t)}{\partial x}
$$

Then power spectral density of the power flow can be obtained

$$
S_{I}(x, \omega)=E_{0} I_{0} \cdot \lim _{T \rightarrow+\infty} \frac{1}{T} E\left\{R e\left[\frac{\partial^{3} U^{*}}{\partial x^{3}} V-\frac{\partial^{2} U^{*}}{\partial x^{2}} \frac{\partial V}{\partial x}\right]\right\}
$$

where $U$ and $V$ are the finite Fourier transforms of the displacement and velocity responses of the beam respectively. Using the mobility function defined in Eq. (18), Eq. (28) can be derived finally as

$$
S_{I}(x, \omega)=E_{0} I_{0} \int_{0}^{L} \int_{0}^{L} R e\left[\frac{\mathrm{j}}{\omega}\left(\frac{\partial^{3} Y^{*}(x, r, \omega)}{\partial x^{3}} Y(x, s, \omega)-\frac{\partial^{2} Y^{*}(x, r, \omega)}{\partial x^{2}} \frac{\partial Y(x, s, \omega)}{\partial x}\right) S_{f}(r, s, \omega)\right] \mathrm{d} r \mathrm{~d} s
$$

The time averaged intensity in a beam subject to random excitation is obtained through the integration of the spectral density of the power flow over frequency domain, as $I(x)=(1 / 2 \pi) \int_{-\infty}^{+\infty} S_{I}(x, \omega) \mathrm{d} \omega$.

\section{Validation of random EFA for a single beam}

In this section, the validity of random EFA in predicting energy density and intensity for beam structures is to be investigated from low to high frequencies by comparing approximate results evaluated using it with exact solutions from modal analysis.

For the first verification case, a simply supported beam subject to distributed broadband excitation is considered. The distributed loading is applied on the central region of the beam, as shown in Fig. 1. The acting region is half of the beam length. The force is assumed to be spatially uncorrelated, which has the cross-spectrum function as

$$
S_{f}\left(x_{1}, x_{2}, \omega\right)=S_{f}(\omega) \delta\left(x_{1}-x_{2}\right), \quad\left(L / 4 \leqslant x_{1}, x_{2} \leqslant 3 L / 4\right)
$$




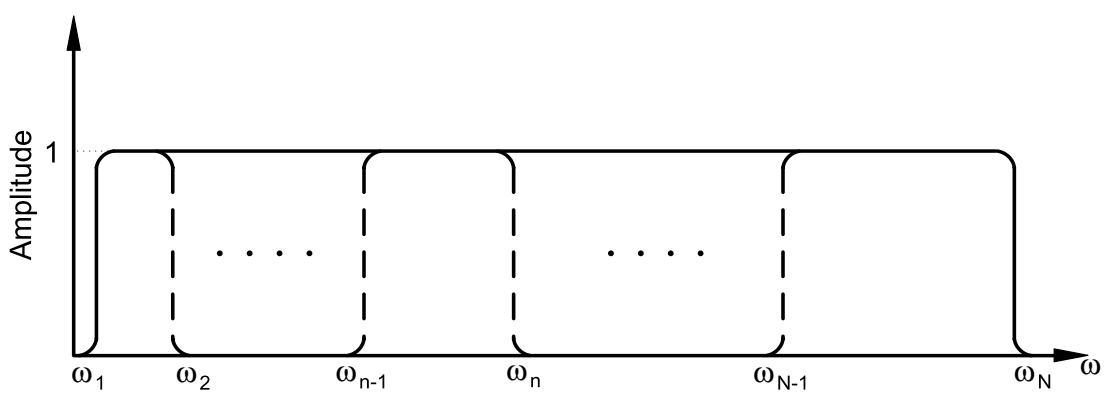

Fig. 2. Auto-spectrum of the random force.
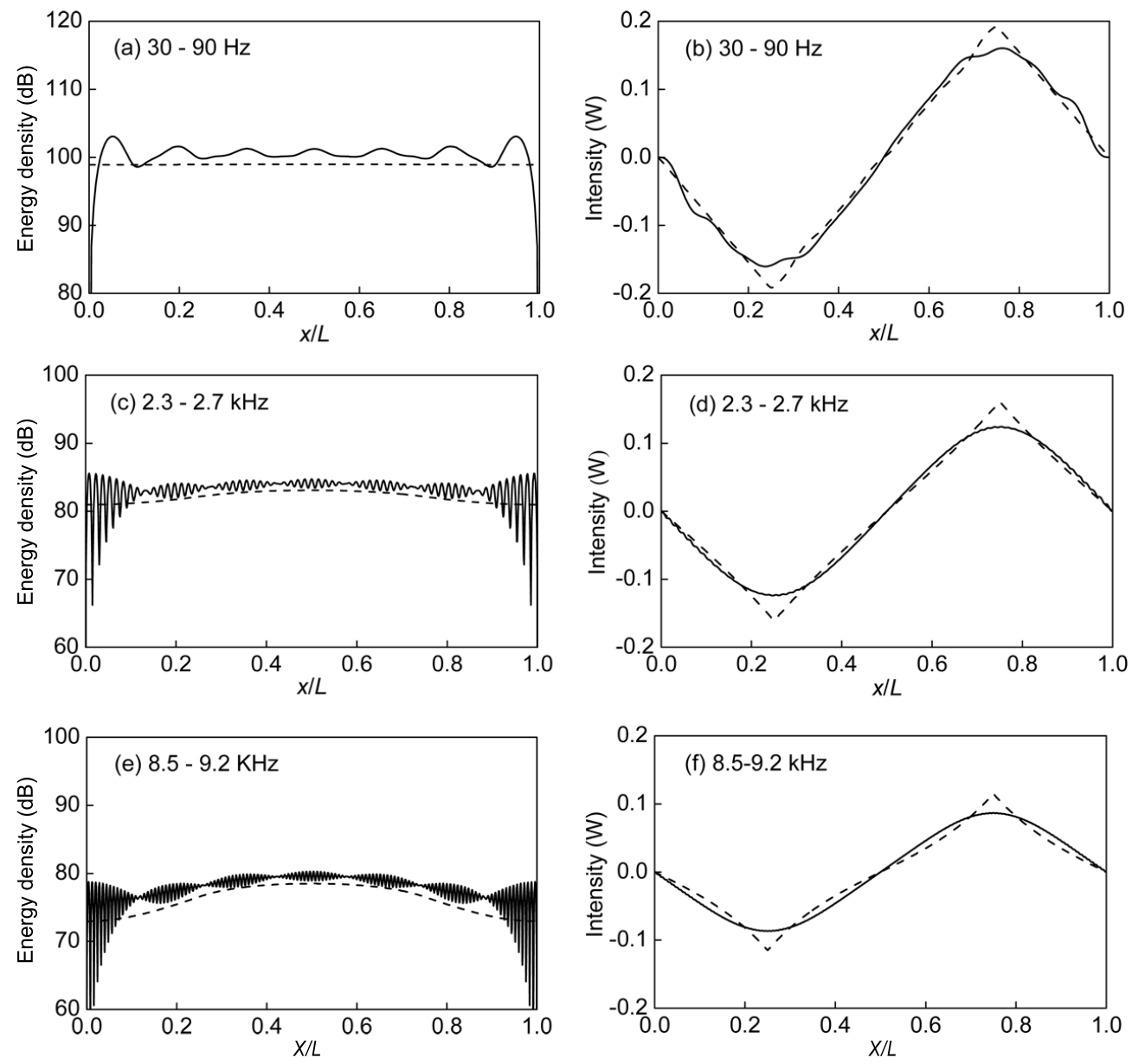

Fig. 3. Response of the beam when distributed force is applied. - exact solution from modal analysis; - - -, random EFA approximation. (a), (c), (e): energy density distribution in different frequency bands; (b), (d), (f) intensity in different frequency bands. The reference energy is $1 \times$ $10^{-12} \mathrm{~J} \mathrm{~m}^{-1}$.

and $S_{f}(\omega)$ is assumed to have flat and broadband spectral distribution as shown in Fig. 2, which can be considered as being composed of many band-limited components. Beam properties are listed in Table 1. For three bandlimited white noise cases, of which frequency ranges are chosen to be $30-90 \mathrm{~Hz}, 2.3-2.7 \mathrm{kHz}$ and $8.5-9.2 \mathrm{kHz}$ with bandwidth of $60 \mathrm{~Hz}, 500 \mathrm{~Hz}$ and $700 \mathrm{~Hz}$ respectively, the approximate and exact results of the energy density and intensity are calculated. Each case of frequency band covers about 5 modes of the beam. For the numerical 

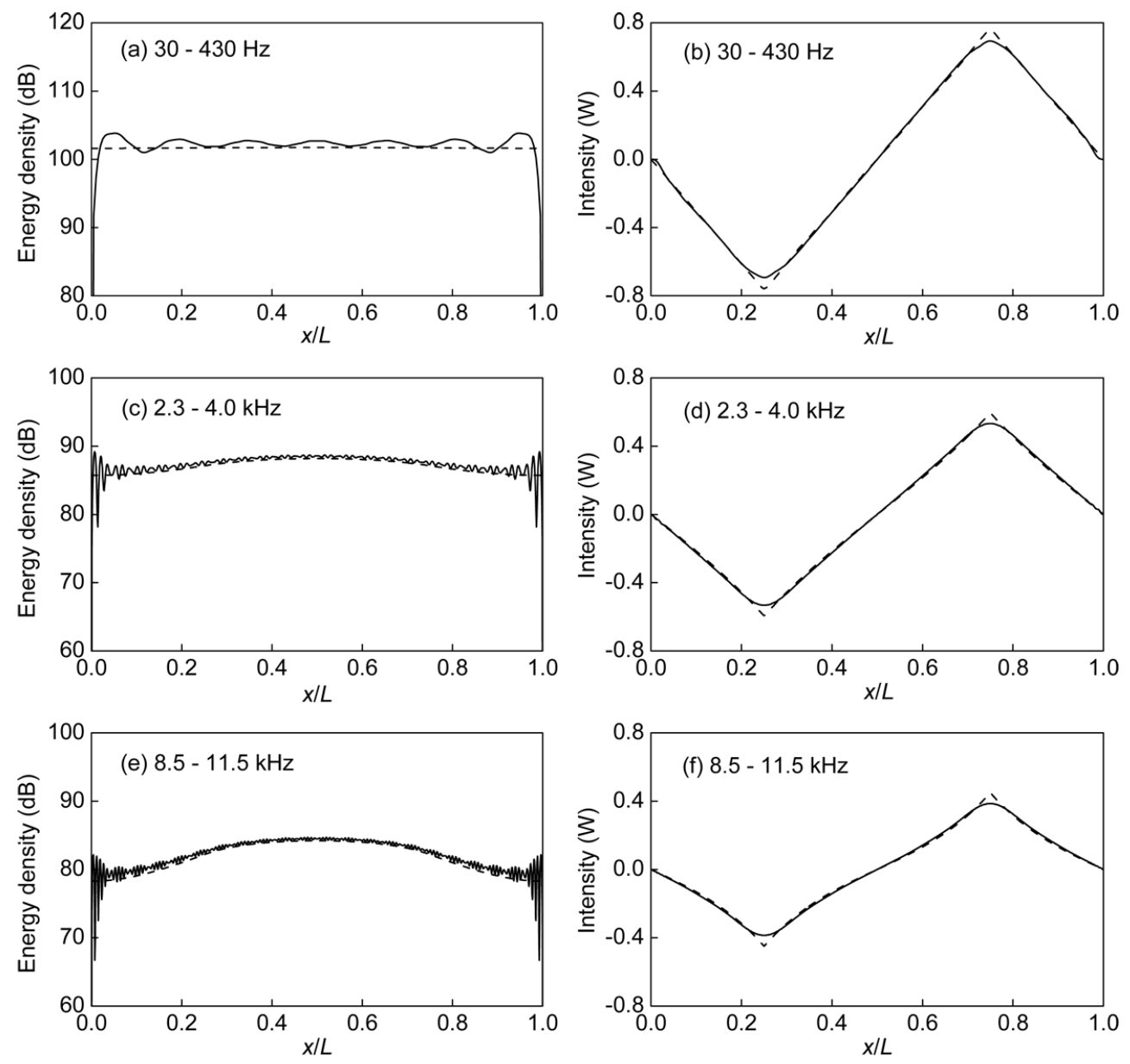

Fig. 4. Response of the beam when distributed force is applied. - , exact solution from modal analysis; - - -, random EFA approximation. (a), (c), (e): energy density distribution in different frequency bands; (b), (d), (f) intensity in different frequency bands. The reference energy is $1 \times$ $10^{-12} \mathrm{~J} \mathrm{~m}^{-1}$.

implementation of the analytical solution of random EFA for a single beam, the exact mobility function is utilized in computing the power input spectrum, and the energy density spectrum is evaluated at 500 discrete frequencies in each frequency band to ensure convergence of the solution. A significant greater number of modes were chosen for each frequency band than number of modes covered in the band to ensure exact results from modal method converged.

Figure 3 displays the comparison between the energy density and intensity predictions from random EFA and modal analysis approach. It is observed that for each frequency band, the energy density evaluated by random EFA is about $2 \mathrm{~dB}$ lower than the exact solutions along the beam. Considering that the three frequency bands have different bandwidths, the nearly same error involved in each case implies that it is the mode count of the band not the bandwidth that has the direct effect on the prediction accuracy of random EFA. The near field effect appears at both boundaries of the beam, where relatively greater discrepancy between the approximate and exact results is observed. The global value of the energy response is found to drop dramatically in high frequency, which is caused by the much greater energy dissipation due to the structural damping in that frequency range. The approximate and exact intensity solutions exhibit the same pattern from low to high frequencies, as shown in Fig. 3(b), (d), and (f), however, they do not correlate well with each other.

When the bandwidth for each case is widened to cover about 20 modes of the beam respectively, i.e., the frequency ranges of the three bands change to $30-430 \mathrm{~Hz}, 2.3-4.0 \mathrm{kHz}$ and $8.5-11.5 \mathrm{kHz}$ respectively, the corresponding approximate and exact solutions for energy density and intensity are shown in Fig. 4. It is found that errors involved 

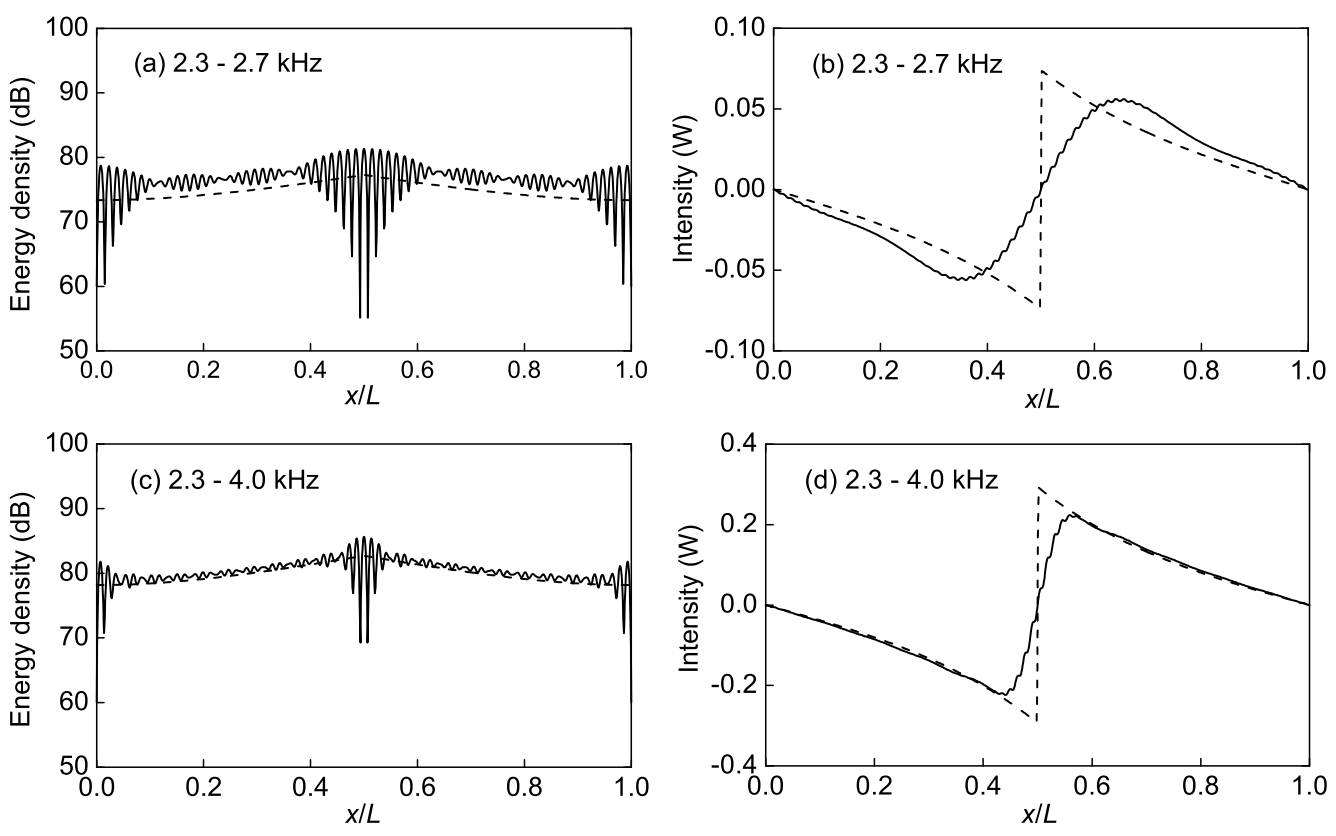

Fig. 5. Response of the beam when a concentrated force is applied at the beam center. - , exact solution from modal analysis; - - -, random EFA approximation. (a), (c): energy density distribution for different bandwidth; (b), (d): intensity for different bandwidth. The reference energy is 1
$\times 10^{-12} \mathrm{~J} \mathrm{~m}^{-1}$.

with random EFA are very slight with respect to energy density, and the approximate predictions of intensity correlate well with the exact ones. This indicates that random EFA prediction approaches the exact response if sufficient modes are included in the frequency band, and this holds nearly over the whole frequency range. Two reasons account for this characteristic of random EFA. The first is associated with the near field effect. It is known that the near field terms of displacement response are neglected in the derivation of the EFA governing equation, causing prediction error when the method is applied. Comparing the exact energy solutions for the three frequency bands in Fig. 4 with their counterparts in Fig. 3, it is observed that the scope of the near field at beam boundaries decreases as more modes are included in the frequency band, and this reduces the error due to the neglect of near field effects in random EFA. The second reason is associated with the concept of space averaging adopted in random EFA. Energy solution provided by the method is the locally averaged over a wavelength result of the exact response, thus the approximate result is expected to approach the exact one when the latter fluctuates with constant amplitude in spatial range comparable with wavelength. When fewer modes are included in the frequency band, the exact energy density exhibits fluctuation with amplitude varying significantly in space as shown in Fig. 3, which makes the approximate result from random EFA deviate greatly from the exact response. When frequency band covers more modes, it is shown in Fig. 4 that the exact energy response fluctuates in space with little change in amplitude, making the approximate result close to the exact solution.

For the second verification case, a simply supported beam excited by a concentrated random force at the center is considered. Auto-spectrum of the force is the same as that described in Fig. 2. The characteristic of random EFA that its prediction approaches the exact result when mode count of the frequency band is sufficiently large also holds for this loading situation, and only the comparison between approximate and exact solutions for frequency bands of $2.3-2.7 \mathrm{kHz}$ and $2.3-4.0 \mathrm{kHz}$ are presented in Fig. 5. The two phenomena associated with the boundary near field effect and spatial fluctuation of the exact response are also observed in this figure, besides, new features can be found with respect to the exact solution. In Figs 3 and 4, no obvious near filed effect appears at the acting region of the distributed force, whereas when concentrated force is applied, near field effect occurs near the acting position of the force as shown in Fig. 5. Moreover, the approximate intensity is seen not to correlate with exact one as well as the case for distributed excitation in the same frequency range. Though the scope of the near field at the driving position decreases as frequency band becomes broader, it is expected that random EFA gives less accurate prediction for concentrated excitation than for distributed excitation when the same frequency range is considered. 

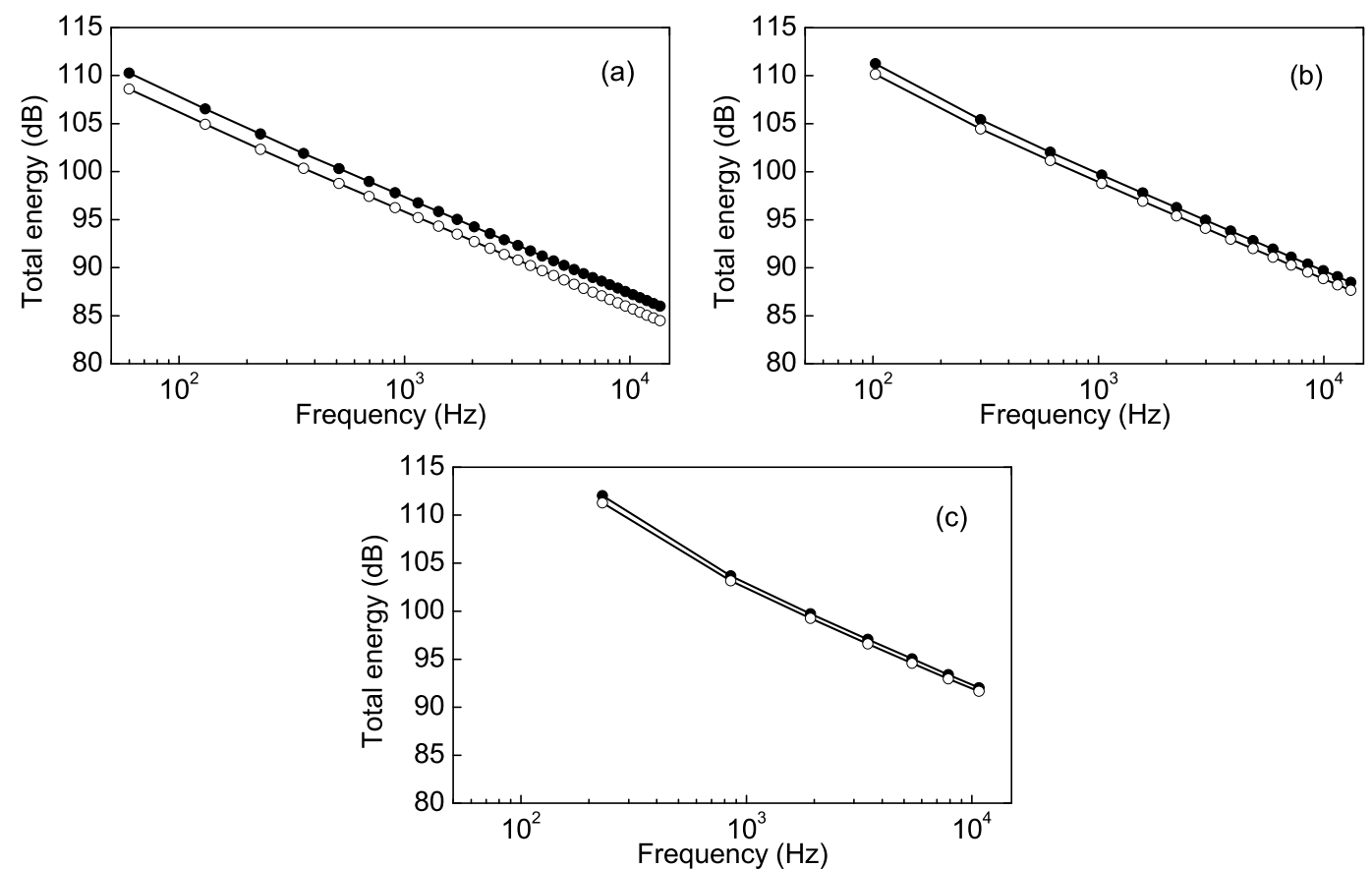

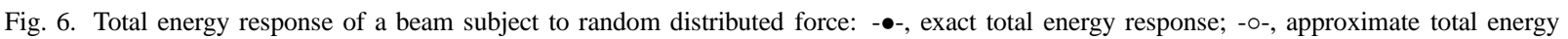
response. Each frequency band contains: (a) 5 modes; (b) 10 modes; (c) 20 modes. The reference energy is $1 \times 10^{-12} \mathrm{~J}$.

In order to further investigate the effect of the mode count of frequency band on the prediction accuracy of random EFA, the verification calculation for distributed and concentrated loadings are performed over the whole frequency range when frequency band with different mode counts are considered. Total energy responses of the beam in each frequency band are obtained for both approximate and exact solutions by integrating the energy density over the beam length. Figures 6 and 7 present the comparisons between the approximate and exact total energy responses for the two cases of distributed and concentrated loadings respectively. For the case of distributed excitation, it is shown in Fig. 6 that when each frequency band has the same mode count, the estimation error of the total energy using random EFA nearly keeps constant over the whole frequency range. For the situation of concentrated excitation, similar characteristic can be found when each frequency band covers adequate number of modes, however, when mode count of the frequency band is low, total energy response estimated by random EFA is unreliable as indicated in Fig. 7(a).

\section{Conclusions}

The validity of random EFA for beam structures is investigated through comparisons between results of approximate and exact responses with respect to both energy density and intensity. The Fourier transform technique is used to find the analytical solution to the random EFA governing equation, and the exact average energy density and intensity of a beam under random excitation is derived through mode superposition method. Validity investigation of random EFA is carried out from low to high frequencies, and three characteristics of the method being applied to a single beam are identified:

(1) The random EFA prediction approaches the exact result if the mode count of the frequency band is sufficiently large, and this holds nearly over the whole frequency range. The verification cases imply that random EFA can provide rather reliable results when the frequency band covers more than 5 modes.

(2) Due to the near field effects caused by concentrated forces at driving positions, for the same frequency band, random EFA gives less accurate energy estimation for concentrated excitation than for distributed excitation. 

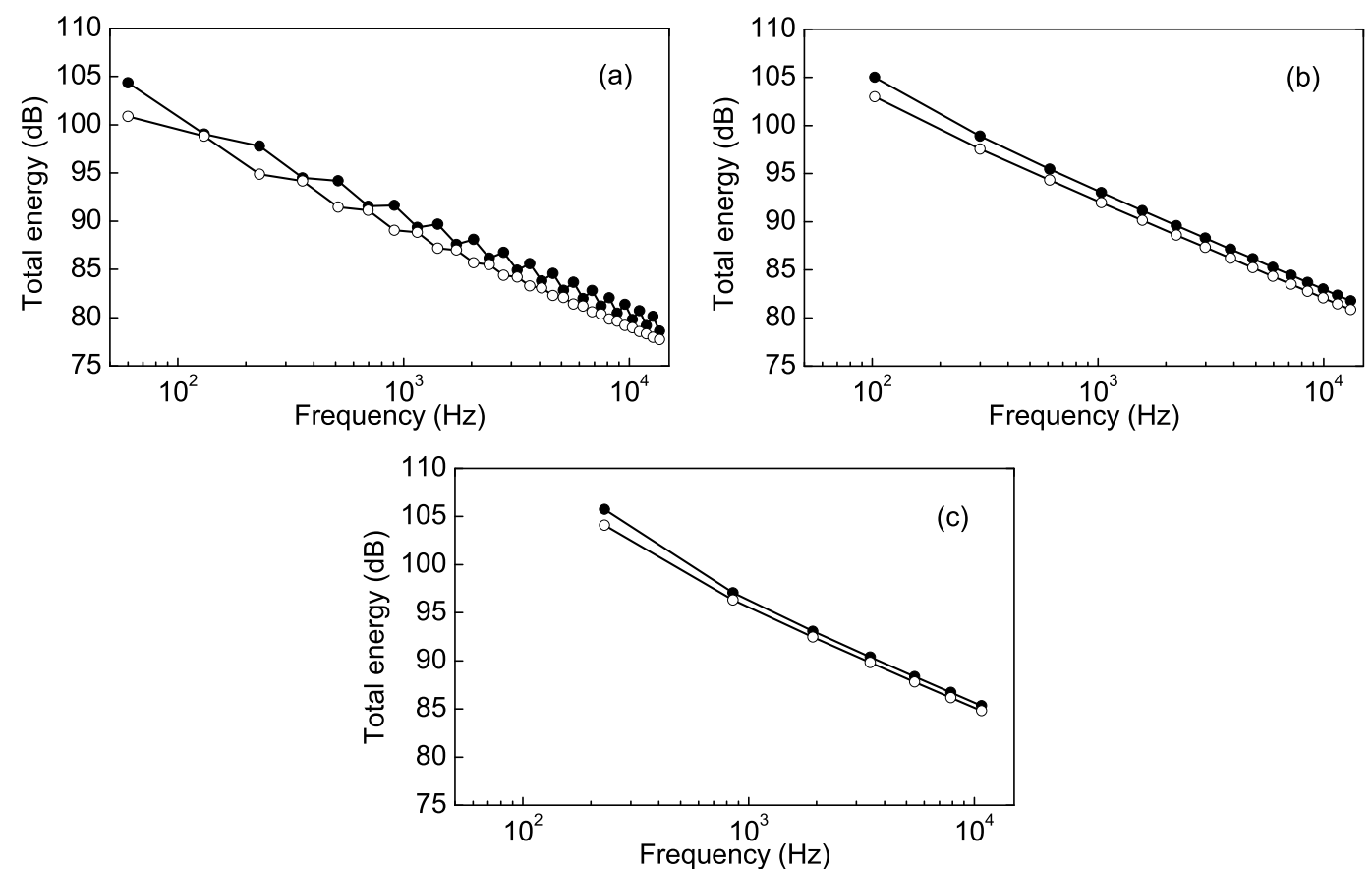

Fig. 7. Total energy response of a beam subject to a concentrated force at the beam center: - $\bullet-$, exact total energy response; - --, approximate total energy response. Each frequency band contains: (a) 5 modes; (b) 10 modes; (c) 20 modes. The reference energy is $1 \times 10^{-12} \mathrm{~J}$.

(3) For distributed excitation, when each frequency band has the same mode count, the estimation error of the total energy response of beam structures using random EFA nearly keeps constant over the whole frequency range, and this also holds for concentrated excitation when each frequency band covers adequate number of modes.

\section{Acknowledgements}

The authors are grateful for the supports received from the National Natural Science Foundation of China (No. 10972137) and the National High-tech Research and Development Foundation of China (No. 2009AA04Z419).

\section{References}

[1] C. Antonio and S. Aldo, Is it possible to treat confidentially SEA the wolf in sheep's clothing? Mechanical Systems and Signal Processing 20 (2006), 1372-1399.

[2] O.M. Bouthier and R.J. Bernhard, Simple models of the energetics of transversely vibrating plates, Journal of Sound and Vibration 182 (1995), 149-164.

[3] O.M. Bouthier and R.J. Bernhard, Simple models of energy flow in vibrating membranes, Journal of Sound and Vibration 182 (1995), $129-147$.

[4] A. Carcaterra and L. Adamo, Thermal analogy in wave energy transfer: Theoretical and experimental analysis, Journal of Sound and Vibration 226 (1999), 253-284.

[5] P.E. Cho, Energy flow analysis of coupled structures, PhD Dissertation, Purdue University, 1993.

[6] P.E. Cho and R.J. Bernhard, Energy flow analysis of coupled beams, Journal of Sound and Vibration 211 (1998), 593-605.

[7] F.J. Fahy, Statistical energy analysis: a critical overview, Philosophical Transactions of the Royal Society: Physical and Engineering Sciences 346 (1994), 431-447.

[8] F. Han, L.G. Mongeau and R.J. Bernhard, Energy flow analysis of beams and plates for random distributed loading, Journal of Fluids and Structures 12 (1998), 315-333.

[9] F. Han, R.J. Bernhard and L.G. Mongeau, Energy flow analysis of vibrating beams and plates for discrete random excitations, Journal of Sound and Vibration 208 (1997), 841-859. 
[10] M.N. Ichchou, A. Le Bot and L. Jezequel, Energy models of one-dimensional, multi-propagative systems, Journal of Sound and Vibration 201 (1997), 535-554.

[11] L. Ji and B.R. Mace, Statistical energy analysis modelling of complex structures as coupled sets of oscillators: Ensemble mean and variance of energy, Journal of Sound and Vibration 317 (2008), 760-780.

[12] R.S. Langley and K.M. Khumbah, Prediction of high frequency vibration levels in built-up structures by using wave intensity analysis, Proceedings of the 36th AIAA/ASME/ASCE/AHS/ASC Structures, Structural Dynamics, and Materials Conference, LA, USA, 1995, 3087-3094.

[13] R.S. Langley and A.N. Bercin, Wave intensity analysis of high frequency vibrations, Philosophical Transactions of the Royal Society: Physical and Engineering Sciences 346 (1994), 489-499.

[14] R.S. Langley, On the vibrational conductivity approach to high frequency dynamics for two-dimensional structural components, Journal of Sound and Vibration 182 (1995), 637-657.

[15] Y. Lase, M.N. Ichchou and L. Jezequel, Energy flow analysis of bars and beams: theoretical formulations, Journal of Sound and Vibration 192 (1996), 281-305.

[16] R.H. Lyon and R.G. DeJong, Theory and application of statistical energy analysis, Butterworth-Heinemann, Boston, 1995.

[17] B.R. Mace, Statistical energy analysis: coupling loss factors, indirect coupling and system modes, Journal of Sound and Vibration 279 (2005), 141-170.

[18] B.R. Mace and L. Ji, The statistical energy analysis of coupled sets of oscillators, Proceedings of the Royal Society A: Mathematical, Physical and Engineering Sciences 463 (2007), 1359-1377.

[19] S.-H. Seo, S.-Y. Hong and H.-G. Kil, Power flow analysis of reinforced beam-plate coupled structures, Journal of Sound and Vibration 259 (2003), 1109-1129.

[20] A. Sestieri and A. Carcaterra, Space average and wave interference in vibration conductivity, Journal of Sound and Vibration 263 (2003), $475-491$.

[21] N. Vlahopoulos and X. Zhao, An investigation of power flow in the mid-frequency range for systems of co-linear beams based on a hybrid finite element formulation, Journal of Sound and Vibration 242 (2001), 445-473.

[22] N. Vlahopoulos, G. Zhang and J. He, Combining an energy boundary element with an energy finite element analysis for airborne noise simulations, 2007 SAE Noise and Vibration Conference, Traverse City, Michigan, 2007, SAE Paper No. 2007-01-2178.

[23] N. Vlahopoulos, S. Li and J. Sun, Combining energy boundary element and energy finite element simulations for airborne noise analysis, in Proceedings of 2006 ASME International Mechanical Engineering Congress and Exposition, Chicago, USA, 2006, IMECE Paper 2006-14114.

[24] J.C. Wohlever and R.J. Bernhard, Mechanical energy flow models of rods and beams, Journal of Sound and Vibration 153 (1992), 1-19.

[25] J. You, G. Meng and H.-G. Li, Random energy flow analysis of coupled beam structures and its correlation with SEA, Archive of Applied Mechanics (2009), DOI: 10.1007/s00419-009-0395-x.

[26] G. Zhang and N. Vlahopoulos, Validation of an EFEA formulation for computing the vibrational response of complex structures, 2007 SAE Noise and Vibration Conference, Michigen, USA, 2007, SAE Paper No. 2007-01-2324.

[27] W. Zhang, N. Vlahopoulos and K. Wu, An energy finite element formulation for high-frequency vibration analysis of externally fluid-loaded cylindrical shells with periodic circumferential stiffeners subjected to axi-symmetric excitation, Journal of Sound and Vibration $\mathbf{2 8 2}$ (2005), 679-700.

[28] W. Zhang, A. Wang, N. Vlahopoulos and K. Wu, High-frequency vibration analysis of thin elastic plates under heavy fluid loading by an energy finite element formulation, Journal of Sound and Vibration 263 (2003), 21-46.

[29] X. Zhao and N. Vlahopoulos, A basic hybrid finite element formulation for mid-frequency analysis of beams connected at an arbitrary angle, Journal of Sound and Vibration 269 (2004), 135-164. 

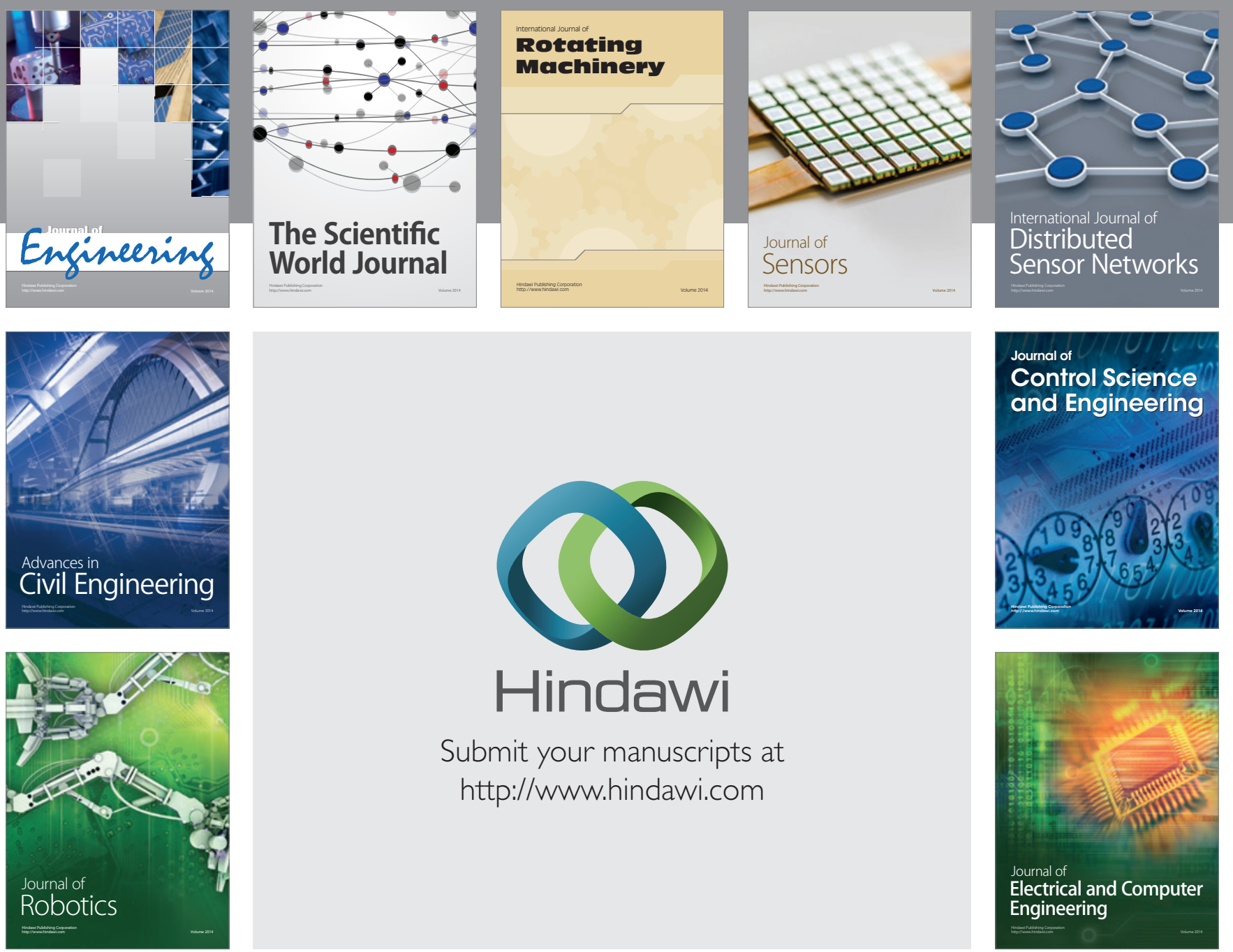

Submit your manuscripts at

http://www.hindawi.com
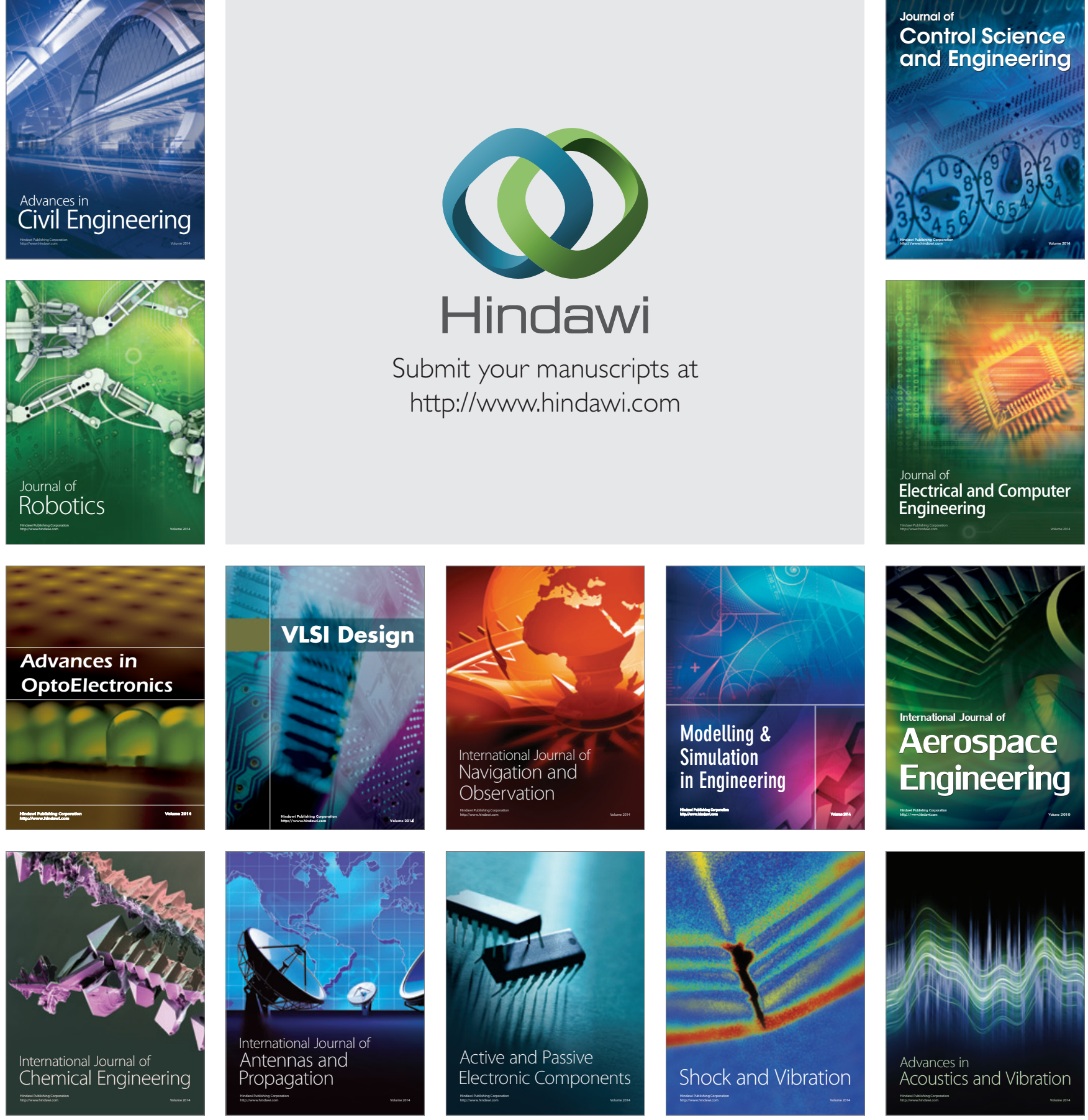